\title{
Foundation Analysis of Emotion Model for Designing Learning Companion Agent
}

\author{
Chien-Chih Huang, Rita Kuo ${ }^{1}$, Maiga Chang ${ }^{2}$, Jia-Sheng Heh \\ Dept. of Information and Computer Engineering, Chung-Yuan Christian Univ. \\ ${ }^{1}$ Dept. of Electronic Engineering, Chung-Yuan Christian Univ. \\ ${ }^{2}$ Dept. of Special Education, Chung-Yuan Christian Univ. \\ lupin@mcsl.ice.cycu.edu.tw,rita@mcsl.ice.cycu.edu.tw,maiga@ms2.hinet.net, \\ jsheh@ice.cycu.edu.tw
}

\begin{abstract}
This paper proposes the architecture of learning companion agent with facial expression of emotion. Based on ABC and ToK architecture, the emotion agent architecture contains five modules to realize the interaction in the world. A particular part of this research is the transition between emotion space in emotion module and facial expression space in facial expression module. Using this transition and those five modules, a small experiment website with emotion agent demonstrates the facial expression of the agent and its emotion tracks.
\end{abstract}

\section{Introduction}

Intelligent agents are wild used assistant tools in different domains of computer applications. In CAI instruments, some distance learning systems propose agents for managing system files [22], notifying grades in on-line quiz system [18], diagnosing problems in problem solving system [7][8][11] and so on. However, most of the agents have only functions of dealing problem but lacks of emotion expressions. Recent researches show that if the intelligent has facial emotion expressions, learners would feel the agent more reliable for them. [1] [2] [5]

The studies of emotion and face expression have been applied in many areas. In counseling education, tutors often use various types of facial-mask expression for students understanding emotion and themselves. Artificial Intelligent domain also integrates autonomic emotion into AI systems in order to make robots more believable. [4] Another emotion application in AI is facial identification, which make robots have ability to recognize human emotion. [16]
How to make intelligent agents have autonomic emotion becomes another interesting research topic for cognitive and computer scientists. This paper proposes an architecture of emotion agent as learning companion. Section 2 mentions some important researches about emotion. The analysis of the architecture of emotion agent is described in section 3 . Section 4 gives a sample site to realize the idea of this paper. A brief conclusion and future work exists in section 5 .

\section{Emotion}

For a long time, emotion is not a popular topic of the research but has strong relation with daily life. Izard has indicated that infants reveal their various emotions at different times over the first two years. [12] The related research domain of emotion includes physiology, cognitive science, behavioral science, philosophy, and phenomenology. [24]

Furthermore, some researchers analyze the types of basic emotional model. OCC emotional model is one of pilot studies in emotion analysis and proposed twenty-two basic emotions. [17] There are also different kinds of classification in emotion model presented from other researchers, such as Gomi, Kort, Picard and so on. [10][14][19]

Emotion expression means the representation of feeling by external activities. Non-linguistic emotion expression is one of its research topics. Facial expression is one kind of non-linguistic one for connecting internal emotion by using facial features and can be easily recognized in different races begins at three year-old. [15] [23] For example, Yuasa and his colleagues use only simply types of eyebrow and mouth to construct facial expression and design 
interface agent for users having ability to send their emotions to others. [25]

After discussing some elements of emotion, theoretical system definition of emotion is also an important architecture of emotion analysis. In 1884, James first discuss the connection among stimulus environments, physiological changes, and emotional experience, which Lange also proposed similar idea in 1885. [13] This theory is named as James-Lange theory and is queried by Cannon and Bard in 1927 by proposing Cannon-Bard theory. [6] In 1962, Schachter and Singer emphasized the attribution of emotion and proposed two-factor theory of emotion. [20] [21]

According to the idea of rational-emotive psychology, Dryen use ABC architecture as his emotion theory to explain the structure of emotional environment. [9] ABC architecture has three stages of emotion generation. The process of generating emotion and behavior begins at the activating events (A), which includes the explanations and inferences of the activities. The activity and the beliefs (B) of the activity in people's mind influence the consequence (C) of emotions and behaviors. This architecture can be explained in Figure 1 and be used in the agent model analysis of later section.

This paper use these researches to construct an agent architecture which simulating human emotionchange progress.

\section{Basic Emotion and Face Expression}

\subsection{Emotion Space and Emotion Expression}

To build emotion space for presenting emotion, this research propose four emotion elements according to five basic emotion factors from Yuasa [25] and four common emotions from Kort. [14] According to these two researches and previous studies, four basic emotion factors are happy, sad, cool, and anger. This definition can be written as $S_{\text {emotion }}=\left\{e_{\text {happy }}, e_{\text {sad }}, e_{\text {cool }}\right.$, $\left.e_{\text {anger }}\right\}$. In these four factors, $e_{\text {happy }}$ and $e_{\text {sad }}, e_{\text {cool }}$ and $e_{\text {anger }}$ are determined as inverted emotions. For this reason, the four factors can build a basic emotion space as Figure 2 shows.

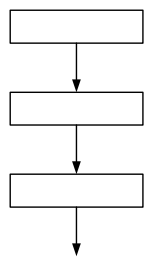

Figure 1. $A B C$ architecture

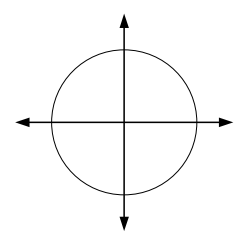

Figure 2. Basic emotion space
In this definition, each item in emotion space can be denoted as $\left[x_{i}, y_{i}\right]$ and is constrained in a unit circle (which radius is 1). According to this restriction, all emotion in emotion space can be expressed in polar coordinates

$$
e_{i}=\left[x_{i}, y_{i}\right]=\left[r_{i}, \theta_{i}\right]=\left[\sqrt{x_{i}^{2}+y_{i}^{2}}, \operatorname{atan} \frac{y_{i}}{x_{i}}\right] \quad x_{i} \neq 0,0<r_{i} \leq 1
$$

$r_{i}$ can be considered as the intensity of emotion and $\theta_{i}$ can be defined as angle of emotion. Using this definition can make emotion vector have ability to be operate in the following analysis. First of all is emotion reinforcement, which means emotion combination. Here define emotion operation

$$
\Phi_{\text {comb }}\left(e_{i}+e_{j}\right)=\left[x_{i}, y_{i}\right]+\left[x_{j}, y_{j}\right]=\left[x_{i}+x_{j}, y_{i}+y_{j}\right]
$$

The intensity of emotion would also be fade away by time, so the next operation of emotion is emotion fade out which of definition is listed below.

$$
\Phi_{\text {fadeout }}\left(e_{i}, \rho_{\text {fade }}\right)=\left[x_{i}-\rho_{\text {fade }} \times \cos \left(\theta_{i}\right), y_{i}-\rho_{\text {fade }} \times \sin \left(\theta_{i}\right)\right]
$$

$\rho_{\text {fade }}$ determines the factor of emotion fade out and be considered by individual and environment.

\subsection{Facial Expression}

After having the definition of emotion space and its operation, the way to express emotion is another point of this paper. Face expression is one of the methods for presenting one's feeling. Simplifying the research of Yuasa, the facial expression space defined in this paper focuses only on eyebrow and mouth. [YNN01] These two elements can be used to construct facial expression space with eyebrow and mouth axes (Figure 4).

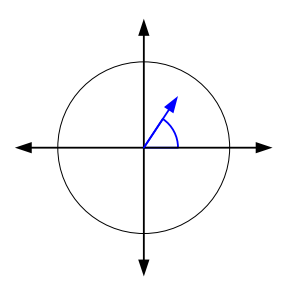

Figure 3. Emotion expression in polar coordinates

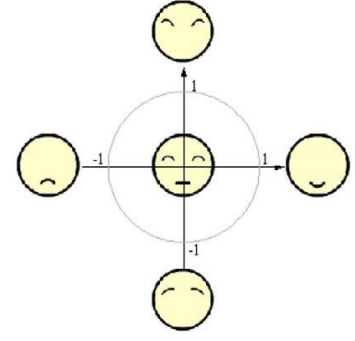

Figure 4. Facial expression space based on eyebrow and mouth
The item in facial expression space can also be formulated in vector. $f_{a}$ means one item in facial expression space and have two dimensions which are mouth-curve $m_{a}$ and eyebrow-curve $b_{a}$. The magnitude of facial expression is

$$
\text { maganitude }\left(f_{a}\right)=\sqrt{m_{a}^{2}+b_{a}^{2}}
$$

Its direction can be written as 


$$
\operatorname{direction}\left(f_{a}\right)=\operatorname{atan}\left(\frac{b_{a}}{m_{a}}\right), \quad m_{a} \neq 0
$$

Corresponding to the four basic emotion factors mentioned in the beginning of section 3, Figure 5 shows the facial expression with only eyebrow and mouth in four basic emotion factors. Mapping the facial emotion space, each emotion factor exists in different quadrant of facial emotion space as shown in Figure 6.

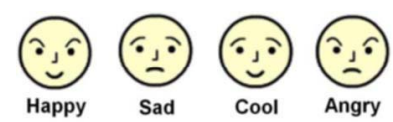

Figure 5. Facial expression of four basic emotion factors

After defining basic emotion factors and facial emotion space, how to translate into each other is the next research point of this paper. Comparing basic emotion space and facial expression space (Figure 6), the angle of two axes in two spaces only have $-\frac{\pi}{4}$ difference. For this reason, the translation from basic emotion space to facial expression space is

$$
\begin{aligned}
& f_{a}=\left[m_{a}, b_{a}\right]=\Phi_{\text {emotion_to_facial }}\left(e_{i}\right)=\left[x_{i} \times \sin \left(\frac{\pi}{4}\right), y_{i} \times \sin \left(\frac{\pi}{4}\right)\right] \text {, or } \\
& \left\{\begin{array}{l}
\text { magnitude }\left(f_{a}\right)=r_{i} \\
\operatorname{direction}\left(f_{a}\right)=\theta_{i}-\frac{\pi}{4}
\end{array}\right.
\end{aligned}
$$

The method of translating facial expression space to basic emotion space can be formulated as

$$
\begin{aligned}
e_{i}= & {\left[x_{i}, y_{i}\right]=\Phi_{\text {facial_to_emotion }}\left(f_{a}\right)=\left[\frac{m_{a}}{\sin (\pi / 4)}, \frac{b_{a}}{\sin (\pi / 4)}\right], \text { or } } \\
& \left\{\begin{array}{l}
r_{i}=\operatorname{magnitude}\left(f_{a}\right) \\
\theta_{i}=\operatorname{direction}\left(f_{a}\right)+\frac{\pi}{4}
\end{array}\right.
\end{aligned}
$$

\subsection{Emotion agent Architecture}

After defining the motion factors and the way of motion expression, the next step of constructing emotion agent is module analysis. In previous researches, Bates with his colleagues proposed ToK architecture as the system architecture of emotion agent. [3] This architecture consists four major parts including sensory module (Sensory routine and Integrated Sense Model), reaction module (Hap), emotion module (Em), and language module (Gump and Glinda).

This paper integrates $\mathrm{ABC}$ architecture into ToK architecture and considers emotion fade out with time. The new architecture contains five major modules as shown in Figure 7.

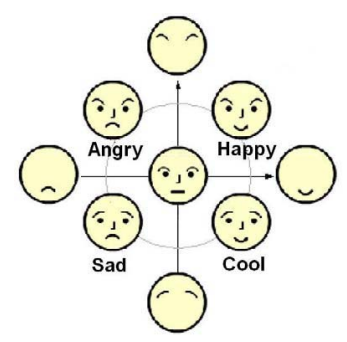

Figure 6. Basic

emotion factor and

emotional expression

space

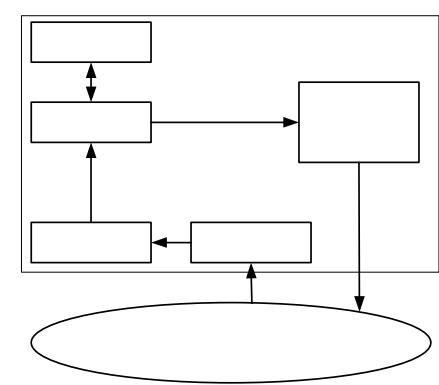

Figure 7. Emotion agent architecture

Sensory module: The sensor module comes from the sensory routine and Integrated Sense Model in ToK architecture. This module is used to gather events (as seem as the Activities of ABC architecture) from world and transform the information into specific format to belief module. Corresponding to the environment in web pages, sensory module should detect users' actions by identifying page types which user click.

Belief module: One event may cause different emotional reaction because of different belief in each individual. The belief module is based on $\mathrm{ABC}$ architecture and gives parameters which corresponding to events to the next module, emotion.

Emotion module: Emotion module includes three functions, which are emotion setting, emotion combination, and emotion fade-out. When the module gets parameters $\overline{b_{k}}$, the corresponding emotion $\overline{e_{k}}$ would be generated. The new generated emotion $\overline{e_{k}}$ would combine the original emotion $\overline{e_{k-1}^{\prime}}$ and use emotion-combination function to generate the integrated emotion $\overline{e_{k}}$. The emotion fade-out function reduces the amplitude of the emotion as time goes on.

Face Expression module: Face Expression module receives the emotion $\overline{e^{\prime}}$ from emotion module. The emotion $\overline{e_{k}^{\prime}}$ would be transformed into facial expression $\overline{f_{k}}$.

Time module: The mechanism of emotion fade-out based on the time scheduling function in time module. This module also records the status of emotion each time.

\section{System Design}

According to the previous analysis of modules in emotion agent, this section implements an emotion agent and one simple website to practice the idea of this paper. Here also follows the sketch in Figure 7 to discuss the world environment and modules' setting. 
World: The website contains two systems, the on-line test system and one small game. Six types of event may be exist in this site, including entering the test page, submitting the test, getting good grades from the test, getting bad grades from the test, playing on-line game on web, and clicking assistant agent. Each event has its event id and would be delivered to sensor module.

Table A. Event types on the web

\begin{tabular}{|l|l|l|}
\hline $\begin{array}{c}\text { Event } \\
\text { ID }\end{array}$ & \multicolumn{1}{|c|}{ Event Name } & \multicolumn{1}{c|}{ Action on Web } \\
\hline A1 & Enter test page & Enter an on-line test page. \\
\hline A2 & Submit test & Finish the test and submit it. \\
\hline A3 & Good grade & Notify the test grade which is good. \\
\hline A4 & Worse grade & Notify the test grade which is worse. \\
\hline A5 & Play game & Start to play the on-line game. \\
\hline A6 & Mouse click & Say hello to the agent \\
\hline
\end{tabular}

Sensor module: The sensor module in the agent only detects actions on the web. The events generated in world would be transformed into sensor module and be sent to belief module.

Belief module: One event may cause different reactions according to different beliefs. The belief module handles switching events to reactions. Table $\mathrm{B}$ displays the mapping table between events and beliefs. When belief module finds the responded Belief ID of the event, the Belief ID would be dispatched to emotion module.

Table B. Switching between events and beliefs

\begin{tabular}{|l|l|l|}
\hline Event ID & \multicolumn{1}{|c|}{ Related Beliefs } & Belief ID \\
\hline \multirow{2}{*}{ A1 } & Be careful of reading the subjects. & B1 \\
\cline { 2 - 3 } & Don't hurry to submit the test. & B2 \\
\hline A2 & Finish the test and feel relaxed. & B3 \\
\hline \multirow{2}{*}{ A3 } & Be praised by parents. & B4 \\
\cline { 2 - 3 } & Get better grade then wondering before. & B5 \\
\hline \multirow{2}{*}{ A4 } & Be blamed by parents. & B6 \\
\cline { 2 - 3 } & The grade should be better. & B7 \\
\hline A5 & This game is fun. & B8 \\
\hline A6 & Say hello to the agent. & B9 \\
\hline
\end{tabular}

Emotion module: The function of emotion module is transform beliefs to emotions. Table $\mathrm{C}$ is the beliefemotion switching table of the example site. Take Belief ID "B1" and "B6" for example. If the emotion module receives " $\mathrm{B} 1$ " from belief module, the new emotion value is $\left[\operatorname{cool}_{k+1}\right.$, happy $\left._{k+1}\right]=\left[\operatorname{cool}_{k}+0.1\right.$, happy $_{k}$. When the Belief ID "B6" was send to emotion module, the new emotion value is $\left[\mathrm{cool}_{k+1}\right.$, happy $\left._{k+1}\right]=\left[\right.$ cool $_{k}$, happy $\left._{k}-(+0.3)\right]$.

Facial expression module: The facial expression module use formulas in section 3 to transform emotion space to facial expression space.
Table C. Mapping table between beliefs and emotions

\begin{tabular}{|c|c|c|c|c|}
\hline $\begin{array}{c}\text { Belief } \\
\text { ID }\end{array}$ & Cool & Angry & Happy & Sad \\
\hline B1 & +0.1 & & & \\
\hline B2 & +0.1 & & & \\
\hline B3 & & & +0.1 & \\
\hline B4 & & & +0.3 & \\
\hline B5 & & & +0.1 & \\
\hline B6 & & & & +0.3 \\
\hline B7 & & & & +0.3 \\
\hline B8 & & & +0.3 & \\
\hline B9 & +0.1 & & +0.3 & \\
\hline
\end{tabular}

By using those environment settings, Figure 8 is a snapshot of the experiment site and the emotion agent. The emotion of the agent would be changed according what action user did on the web pages and the time user spent (Figure 9).

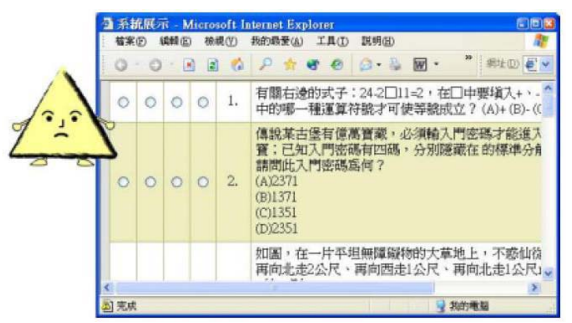

Figure 8. Snapshot of the experiment environment and emotion agent

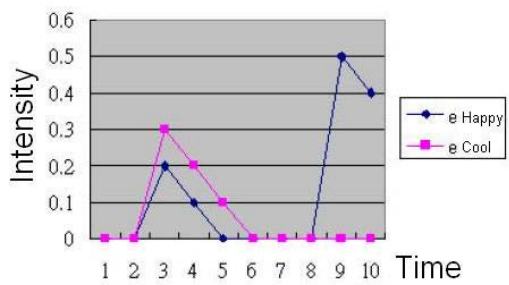

Figure 9. Emotion progress of the emotion agent

\section{Conclusion}

This paper proposed a way to express emotion of the emotion agent. First, the emotion space is defined to construct the basic emotion of the agent. After considering the elements of the emotion, the way to transform emotion to expression is the next step. Facial expression is the presenting way of the agent and has converting relation from emotion space.

The architecture of emotion agent module is also proposed in this study. Five major modules exist in the architecture, which based on ABC and ToK architectures. In the end of the paper, a small example demonstrates the website with emotion agent and its emotion tracks. 
Although this research only proposes a simple way to express agent emotion of simulating human emotion, how to use this architecture to help students in the learning process is another issue to discuss. How to make belief module more reliable is another interesting topic in this paper.

\section{References}

[1] Amy L. Baylor, Suzanne J. Ebbers (2003), "The Pedagogical Agent Split-Persona Effect: When Two Agents are Better than One," World Conference on Educational Multimedia, Hypermedia \& Telecommunications (ED-Media 2003), Honolulu, Hawaii, USA, Jun. 23-28, 2003, pp.459-462

[2] Amy L. Baylor, Yanghee Kim (2003), "Validating Pedagogical Agent Roles: Expert, Motivator, and Mentor," World Conference on Educational Multimedia, Hypermedia \& Telecommunications (ED-Media 2003), Honolulu, Hawaii, USA, Jun. 2328, 2003, pp.463-466

[3] Bates, J., Loyall, A., and Reilly, W. (1992). "Integrating reactivity, goals, and emotion in a broad agent," Proceedings of the Fourteenth Annual Conference of the Cognitive Science Society, Bloomington, Indiana, pp.696-701.

[4] Breazeal, C. and Scassellati, B. (1999) "How to build robots that make friends and influence people", Proceedings of the 1999 IEEE/RSJ Int. Conference on Intelligent Robot and Systems.

[5] Amy L. Baylor, Jeeheon Ryu, E. Shen (2003), "The Effects of Pedagogical Agent Voice and Animation on Learning, Motivation and Perceived Persona," World Conference on Educational Multimedia, Hypermedia \& Telecommunications (ED-Media 2003), Honolulu, Hawaii, USA, Jun. 23-28, 2003, pp.452-458

[6] Cannon, W. B. (1927), "The James-Lange theory of emotion: A critical examination and an alternative theory," American Journal of Psychology ;39, pp.10124.

[7] Janie Chang, Maiga Chang and Jia-Sheng Heh (1999), "Applying the Evaluation Model of Problem Solving to Agent-based Instructional System ," Proceedings of the $7^{\text {th }}$ International Conference on Computers in Education, Chiba, Japan, 1999, pp. 141-148

[8] Janie Chang, Maiga Chang, Jeng-Lun Lin and JiaSheng Heh (2000), "Implements a Diagnostic Intelligent Agent for Problem Solving in Instructional Systems," Proceedings of the First IEEE International Workshop on Advanced Learning Technologies, 2000, Palmerston North, New Zealand, pp.29-30, Dec. 4-6, 2000

[9] Windy Dryden (1994), Invitation to Rational-Emotive Psychology, Whurr Pub Ltd.

[10] Gomi, T.; Vardalas, J.; Koh-Ichi Ide (1995) "Elements of artificial emotion", Robot and Human
Communication, pp. 265 -268

[11] Jia-Sheng Heh (1999), "Evaluation Model of Problem Solving," Mathematical and Computer Modelling, Vol.30, 1999, pp.197-211

[12] Izard, C. E. (1982), Measuring emotions in infants and children, New York: Cambridge University Press.

[13] William James (1950), Principles of Psychology, vol.1, Dover Pubns.

[14] Kort, B.; Reilly, R.; Picard, R.W. (2001) "An affective model of interplay between emotions and learning", Proceedings of IEEE International Conference on Advanced Learning Technologies, 6-8 Aug, 2001, pp.43-46

[15] Lindzey, G., Thompson, R. F., and Spring, B. (1988), Psychology ( ${ }^{\text {rd }}$ Ed.), New York: Worth Publishers.

[16] Matsuno, K. Chil-Woo Lee Kimura, S. Tsuji, S. Kansai Electr. Power Co. Inc. (1995) "Automatic recognition of human facial expressions", Proceedings of Fifth International Conference on Computer Vision, 20-23 June 1995, pp. 352-359.

[17] A. Ortony, G. Clore, A. Collins (1988) The Cognitive Structure of Emotions, Cambridge University Press

[18] A. Okada, H. Tarumi and Y. Kambayashi (2000), "Real-time quiz functions for dynamic group guidance in distance learning systems," Proceedings of the First International Conference on Web Information Systems Engineering, 2000, Vol.2, pp.188-195

[19] Picard, R.W.; Vyzas, E.; Healey, J. (2001) “Toward Machine Emotional Intelligence-Analysis of Affective Physiological State", Pattern Analysis and Machine Intelligence, IEEE Transactions, pp.11751191 vol.23

[20] Schachter, S. (1964), "The interaction of cognitive and physiological determinants of emotional state," In L. Berkowitz (ed.), Advances in experimental social psychology, Volume 1, pp.49-80, New York: Academic Press

[21] Schachter, S. and Singer, J. E. (1962), "Cognitive, social and physiological determinants of emotional states," Psychological Review, 69, pp.379-399

[22] A. Shimano and H. Kuramae (2000), "Design and construction of educational computer system using self-maintenance system for files and user identification agent," Proceedings of the 9th IEEE International Workshop on Robot and Human Interactive Communication, 2000. RO-MAN 2000, pp.23-28

[23] Stifer, C., and Fox, N. (1986), "Preschool children and ability to identify and lable emotions," Journal of Nonverbal Behavior, 10, pp.255-266

[24] Strongman, K. T. (1987), The Psychology of Emotion $\left(3^{\text {rd }}\right.$ ed.), John Wiley \& Sons

[25] Yuasa, M.; Yasumura, Y.; Nitta, K. (2001) "A negotiation support tool using emotional factors", IFSA World Congress and 20th NAFIPS International Conference, pp.2906 -2911 vol.5 